\title{
Plasmatic ADAMTS-13 metalloprotease and von Willebrand factor in children with cyanotic congenital heart disease
}

\author{
R.P.S. Soares ${ }^{1}$, S.P. Bydlowski ${ }^{2}$, N.M. Nascimento ${ }^{2}$, A.M. Thomaz ${ }^{3}$, \\ E.N.M. Bastos ${ }^{3}$ and A.A. Lopes ${ }^{3}$ \\ ${ }^{1}$ Fundação Pró-Sangue Hemocentro de São Paulo, São Paulo, SP, Brasil \\ ${ }^{2}$ Laboratório de Investigação Médica-31, Faculdade de Medicina, Universidade de São Paulo, São Paulo, SP, Brasil \\ ${ }^{3}$ Instituto do Coração, Faculdade de Medicina, Universidade de São Paulo, São Paulo, SP, Brasil
}

\begin{abstract}
Changes in plasma von Willebrand factor concentration (VWF:Ag) and ADAMTS-13 activity (the metalloprotease that cleaves VWF physiologically) have been reported in several cardiovascular disorders with prognostic implications. We therefore determined the level of these proteins in the plasma of children with cyanotic congenital heart disease (CCHD) undergoing surgical treatment. Forty-eight children were enrolled (age 0.83 to 7.58 years). Measurements were performed at baseline and $48 \mathrm{~h}$ after surgery. ELISA, collagen-binding assays and Western blotting were used to estimate antigenic and biological activities, and proteolysis of VWF multimers. Preoperatively, VWF:Ag and ADAMTS-13 activity were decreased $(65$ and $71 \%$ of normal levels considered as $113(105-129) \mathrm{U} / \mathrm{dL}$ and $91 \pm 24 \%$ respectively, $\mathrm{P}<0.003)$ and correlated $(r=0.39, P=$ 0.0064). High molecular weight VWF multimers were not related, suggesting an interaction of VWF with cell membranes, followed by proteolytic cleavage. A low preoperative ADAMTS-13 activity, a longer activated partial thromboplastin time and the need for cardiopulmonary bypass correlated with postoperative bleeding $(P<0.05)$. Postoperatively, ADAMTS-13 activity increased but less extensively than VWF:Ag (respectively, 2.23 and 2.83 times baseline, $\mathrm{P}<0.0001$ ), resulting in an increased VWF:Ag/ADAMTS-13 activity ratio (1.20 to 1.54, respectively, pre- and postoperative median values, $P=0.0029)$. ADAMTS13 consumption was further confirmed by decreased ADAMTS-13 antigenic concentration $(0.91 \pm 0.30$ to $0.70 \pm 0.25 \mu \mathrm{g} / \mathrm{mL}$, $\mathrm{P}<0.0001)$ and persistent proteolysis of VWF multimers. We conclude that, in pediatric CCHD, changes in circulating ADAMTS-13 suggest enzyme consumption, associated with abnormal structure and function of VWF.
\end{abstract}

Key words: ADAMTS-13; von Willebrand factor; Congenital heart disease; Thrombosis; Cardiac surgery

\section{Introduction}

ADAMTS is an abbreviation for $\underline{a}$ desintegrin and metalloprotease with thrombospondin type 1 motif, ānd corresponds to a family of zinc metalloproteases with desintegrin and protease domains (ADAM-related), cystein-rich regions and a thrombospondin 1 repeat. ADAMTS-13, a member of the family, is synthesized in hepatic stellate cells and endothelial cells (1-3), and is the principal von Willebrand factor (VWF) cleaving protease. Mutations of the ADAMTS-13 gene are associated with the presence of abnormally large VWF multimers in plasma, which constitutes the basis for the thrombotic events that occur in subjects with congenital thrombotic thrombocytopenic purpura. Protease deficiency may also occur in the presence of autoimmune $\operatorname{lgM}$ and $\lg$ inhibitors $(4,5)$.
An imbalance between the circulating levels of VWF and ADAMTS-13 has been reported in a number of acquired diseases such as coronary artery disease and myocardial infarction, peripheral arterial disease, ischemic stroke, preeclampsia, inflammatory bowel disease, and liver cirrhosis (6-9). Elevated plasma VWF antigen (VWF:Ag) and/or decreased ADAMTS-13 activity are associated with negative outcomes of these disorders. In disseminated intravascular coagulation and sepsis, exceedingly high VWF:Ag has been reported in association with a progressive decrease in ADAMTS-13 activity as the severity of the disease increases. This reflects widespread thrombotic microangiopathy generally associated with circulating ultralarge VWF multimers $(10,11)$.

Correspondence: A.A. Lopes, Unidade Clínica de Cardiologia Pediátrica e Cardiopatias Congênitas do Adulto, InCor, HC, FMUSP, Av. Dr. Eneas C. Aguiar, 44, 05403-000 São Paulo, SP, Brasil. Fax: +55-11-2661-5409. E-mail: aablopes@usp.br 
Abnormalities in coagulation parameters and platelet function have been reported in patients with cyanotic congenital heart disease (CCHD). These abnormalities tend to be more pronounced in the perioperative course, particularly in the presence of cardiopulmonary bypass (12-15). Albeit changes in ADAMTS-13 activity and VWF protein levels have been reported in subjects undergoing ON- as well as OFF-pump cardiac surgery $(11,16-18)$, they have not been investigated in the pediatric population with CCHD undergoing surgical palliation or repair. Thus, the present study was planned to analyze the pre- and postoperative abnormalities in circulating ADAMTS-13 and VWF in this setting.

\section{Material and Methods}

Patients, diagnostic data and postoperative events

Pediatric patients with CCHD undergoing cardiac surgery (first operation or reoperation) were enrolled consecutively. Diagnostic data were collected on a hospital basis, one day before surgery, at the Instituto do Coração, Faculdade de Medicina, Universidade de São Paulo, São Paulo, Brazil. Neonates, patients under intensive care and those undergoing emergency cardiac surgery were excluded. Preoperative (baseline) data were registered, including demographic and general laboratory parameters, and the principal diagnosis as established by Doppler-echocardiography and, in some instances, angiography. The need for cardiopulmonary bypass as well as relevant postoperative events (infection, sepsis, bleeding, and fatal outcome) are reported. Relevant postoperative bleeding was defined as $10 \mathrm{~mL} \mathrm{~kg}^{-1} \mathrm{~h}^{-1}$ during the first hour and $5 \mathrm{~mL} \mathrm{~kg}^{-1} \mathrm{~h}^{-1}$ subsequently, with the need for blood product transfusion. The study was approved by the
Scientific and Ethics Committee of the Instituto do Coração and Hospital das Clínicas, Universidade de São Paulo, Brazil (CAPPesq \#07106).

\section{Biochemical analyses}

Peripheral venous blood was collected 1 day before and $48 \mathrm{~h}$ after surgery. Blood was collected [1:10 (v/v) into $3.2 \%$ sodium citrate] for analysis of plasma VWF:Ag and VWF biological activity (VWF:CB), ADAMTS-13 antigen (ADAMTS-13:Ag) and ADAMTS-13 activity. Protease inhibitors were added for the analysis of VWF multimeric structure (19).

Plasma VWF:Ag and ADAMTS-13:Ag were determined by ELISA (Diagnostica Stago, France, and Technoclone, Austria, respectively). Data are reported as $\mathrm{U} / \mathrm{dL}$ and $\mu \mathrm{g} / \mathrm{mL}$, respectively. Plasma $\mathrm{VWF:CB}$ was measured as the ability of the VWF protein to bind to collagen, according to Siekmann et al. (20). An in-house enzyme immunoassay was developed using a peroxidase-conjugated rabbit anti-human VWF polyclonal antibody (Dako Corporation, USA). Data are reported as percent activity relative to normal pooled plasma used in the same assay.

ADAMTS-13 activity was measured by the collagen binding method proposed by Rick et al. (21). Briefly, an inhouse enzyme immunoassay was developed, where plasma was used as the source of both enzyme and substrate. Plasma was processed before (baseline sample) and after dialysis against $1.5 \mathrm{M}$ urea. Diluted plasma samples were added in triplicate to plate wells pre-coated with collagen (Vitrogen, Cohesion Corp., USA). The binding of VWF to collagen was measured using peroxidase-labeled rabbit anti-human VWF polyclonal antibody (Dako Corporation). Plates were analyzed

Table 1. Baseline laboratory data.

\begin{tabular}{lccc}
\hline & Patients $(\mathrm{n}=48)$ & Healthy children* $(\mathrm{n}=9)$ & $\mathrm{P}$ \\
\hline $\mathrm{SpO}_{2}(\%)$ & $80(53-91)$ & $98(95-99)$ & $<0.0001$ \\
Hematocrit $(\%)$ & $47 \pm 7$ & $37 \pm 3$ & $<0.0001$ \\
Hemoglobin $(\mathrm{g} / \mathrm{dL})$ & $15.9 \pm 2.3$ & $12.7 \pm 1.2$ & $<0.0001$ \\
Platelets $\left(\mathrm{x} 10^{9} / \mathrm{L}\right)$ & $313 \pm 114$ & $301 \pm 56$ & 0.8438 \\
Leukocytes $\left(\mathrm{cell} / \mathrm{mm}^{3}\right)$ & $10631 \pm 3613$ & $5667 \pm 1222$ & $<0.0001$ \\
VWF:Ag $(\mathrm{U} / \mathrm{dL})$ & $73(51-159)$ & $113(105-129)$ & $<0.0001^{+}$ \\
VWF:CB $(\%)$ & $48 \pm 21$ & $63 \pm 9$ & $0.0004^{+}$ \\
ADAMTS-13:Ag $(\mu \mathrm{g} / \mathrm{mL})$ & $0.91 \pm 0.29$ & $1.11 \pm 0.09$ & $0.0002^{+}$ \\
ADAMTS-13 activity $(\%)$ & $65 \pm 24$ & $91 \pm 24$ & $0.0029^{+}$ \\
VWF:Ag/ADAMTS-13 activity & $1.17(0.62-4.84)$ & $1.13(1.1-1.69)$ & 0.7427 \\
VWF multimers & $0.44(0.11-0.56)$ & $0.36(0.25-0.55)$ & $0.0081^{+}$ \\
\hline
\end{tabular}

Data are reported as means \pm SD or median and range. ADAMTS-13:Ag = plasma ADAMTS-13 antigenic concentration; $\mathrm{SpO}_{2}=$ peripheral oxygen saturation; VWF:Ag = plasma von Willebrand factor antigenic concentration; VWF:Ag/ADAMTS- 13 activity = ratio of VWF antigenic concentration to ADAMTS-13 activity; VWF:CB = plasma VWF activity reported as collagen binding capacity; VWF multimers $=$ ratio of low molecular weight multimer density to total multimer density of VWF. *Age 5.75 to 7.25 years (median 6.08 years). ${ }^{+}$Differences were found to be significant using both non-parametric and parametric statistics for the appropriate data (MannWhitney test and Student $t$-test). 
on a plate reader at $492 \mathrm{~nm}$. ADAMTS-13 activity was calculated taking into account the residual collagen binding activity after dialysis, and the collagen binding activity in the individual's baseline sample. Data are reported as percent activity.

The multimeric structure of VWF was detected and analyzed by Western blotting as previously described (19). Luminographs were subjected to densitometric analysis, and the relative density of low molecular weight VWF multimers was calculated. Low molecular weight VWF fractions were considered as the five lower bands migrating just above immunoglobulin M (950 kDa, used as a molecular mass marker). The density of these bands was calculated, and the sum was divided by total multimer density.

Biochemical analyses were also performed using samples collected from healthy pediatric subjects, and data are presented as control values. These were children suspected of having mild heart disease, but found to have normal cardiovascular status after diagnostic evaluation.

\section{Statistical analysis}

Data are reported as means and standard deviation or median and range. Comparisons between groups were performed using the Student $t$-test or the Mann-Whitney test. Preoperative and postoperative data were compared using the paired $t$-test or the Wilcoxon test. For correlations, the Spearman coefficient $\left(r_{s}\right)$ was calculated. For variables with Gaussian distribution, linear regression and analysis of covariance (ANCOVA) were used to analyze pre- and postoperative associations. Logistic regression models were constructed to investigate possible associations with postoperative bleeding. A confounder was defined as a second variable causing a significant reduction in the hazard ratio associated with the variable under investigation. Multivariate analysis was carried out by including all variables with a $P$ value of $<0.10$ in univariate and bivariate analysis. In all final procedures, 0.05 was adopted as the level of significance.

\section{Results}

Of 56 consecutive CCHD patients who were candidates for elective pediatric cardiac surgery, 48 were enrolled. Eight children could not be included, as the surgical procedure had to be postponed in view of an acute illness (generally, an acute viral insult). Age ranged from 0.83 to 7.58 years (median 2.54 years) and mean body weight was $11.9 \pm 3.7 \mathrm{~kg}$. Despite decreased peripheral oxygen saturation (Table 1), no patients had hematocrit above $55 \%$. The leukocyte count was significantly elevated compared with healthy pediatric subjects, but there was no evidence of ongoing infectious or inflammatory disorders. The platelet count of the patients was normal, as were blood coagulation tests [activated partial thromboplastin time (APTT) and prothrombin time] (data not shown).

As shown in Table 1, preoperative VWF:Ag and VWF:CB were statistically less in patients. Lower ADAMTS-13:Ag and ADAMTS-13 activity in patients compared to controls (Table 1) were associated with a loss of high molecular mass VWF multimeric protein complexes, resulting in increased density of low molecular mass fractions (Table 1 and Figure 1), which might be interpreted as proteolytic degradation with enzyme consumption. In order to test for possible influences of $A B O$ blood groups on the biochemical parameters (group "O" patients are known to have lower VWF levels compared to "non-O" subjects), VWF and ADAMTS-13 levels were

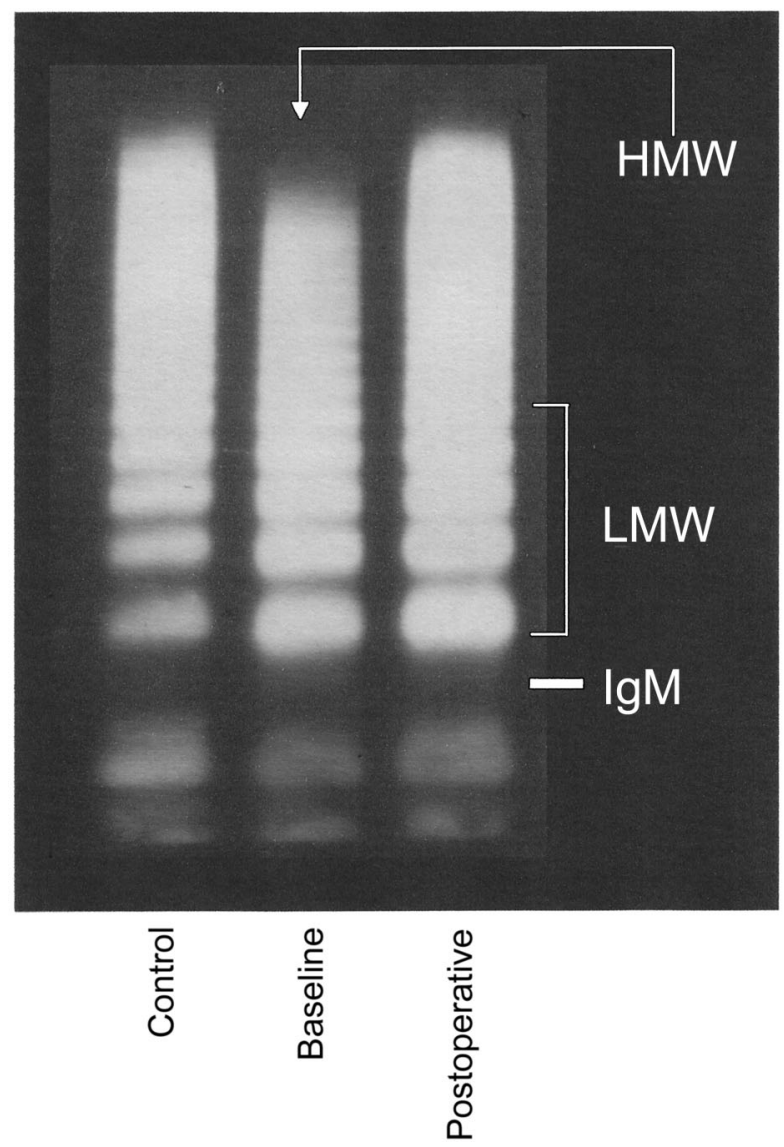

Figure 1. Representative Western immunoblot showing plasma von Willebrand factor multimeric structure (pattern observed in 36 of 48 children with cyanotic congenital heart disease). At baseline, reduction of high molecular weight multimers (HMW), with increased density of low molecular weight fractions (LMW, five bands migrating just above IgM) compared with control plasma (relative optical density of LMW multimers 0.44 (0.11$0.56)$ and $0.36(0.25-0.55)$, respective median value and range in patients and controls, $P=0.0081$ ). Postoperatively, the density of LMW fractions remained high [0.45 (0.17-0.63), $P=0.5360$ versus baseline]. The migration of immunoglobulin $\mathrm{M}$ ( $\mathrm{IgM}$, $950 \mathrm{kDa}$ ) is indicated. 
Table 2. Comparisons of VWF and ADAMTS-13 levels in patients according to ABO blood groups.

\begin{tabular}{lccc}
\hline & Group O patients $(\mathrm{n}=26)$ & Group non-O patients $(\mathrm{n}=22)$ & $\mathrm{P}$ \\
\hline VWF:Ag $(\mathrm{U} / \mathrm{dL})$ & $71(51-159)^{*}$ & $83(51-143)^{+}$ & 0.0216 \\
VWF:CB $(\%)$ & $48 \pm 18$ & $48 \pm 25$ & 0.9446 \\
ADAMTS-13:Ag $(\mu \mathrm{g} / \mathrm{mL})$ & $0.91 \pm 0.28$ & $0.90 \pm 0.31$ & 0.8274 \\
ADAMTS-13 activity $(\%)$ & $62 \pm 20$ & $68 \pm 28$ & 0.4171 \\
\hline
\end{tabular}

Data are reported as means \pm SD or median and range. ADAMTS-13:Ag = plasma ADAMTS-13 antigenic concentration; $\mathrm{VWF}: \mathrm{Ag}=$ plasma von Willebrand factor antigenic concentration; ADAMTS-13 activity = plasma ADAMTS-13 activity concentration; VWF:CB = plasma VWF activity reported as collagen binding capacity. ${ }^{*} \mathrm{P}<0.0001$ and ${ }^{+} \mathrm{P}=0.0004$ versus control level of $113(105-129) \mathrm{U} / \mathrm{dL}$ (Mann-Whitney test)

analyzed in these groups. Table 2 shows that the only statistically significant difference between group "O" and group "non-O" patients was related to VWF:Ag level $(\mathrm{P}=$ 0.0216). However, in both blood groups VWF:Ag level was lower compared to controls. There were no significant associations of VWF and ADAMTS-13 levels with general laboratory data, except for a positive correlation of ADAMTS-13:Ag with peripheral oxygen saturation $\left(r_{\mathrm{s}}=\right.$ 0.39, $\mathrm{P}=0.0059$ ).

Corrective and palliative procedures performed in 48 children included Glenn ( $\mathrm{n}=14)$ and Fontan $(\mathrm{n}=9$ ) operations, total repair of tetralogy of Fallot $(n=11)$ or pulmonary atresia $(n=7)$, Blalock-Taussig anastomoses $(\mathrm{n}=6)$, and pulmonary artery banding $(\mathrm{n}=1)$. Cardiopulmonary bypass was required in 31 cases (duration of 38 to $250 \mathrm{~min}$ ). Peripheral oxygen saturation increased significantly in the entire patient group (Table 3). There were three immediate postoperative deaths associated with low cardiac output and circulatory collapse. None of the 45 remaining patients had any evidence of systemic infection or sepsis. Significant postoperative bleeding occurred in 13 children.

The results obtained at $48 \mathrm{~h}$ postoperatively are presented in Table 3. There was an important (almost three-fold) increase in VWF:Ag, although the density of low molecular weight fractions remained high (Table 3 and Figure 1). VWF:CB increased from baseline. ADAMTS-13 activity increased, albeit not as impressively as VWF:Ag; this resulted in a significant increase in VWF:Ag/ADAMTS-13 activity ratio relative to baseline. ADAMTS-13:Ag actually decreased (Table 3). ADAMTS13 and VWF activities correlated significantly with VWF:Ag at baseline and after surgery (Figure 2). Lowest preoperative levels of ADAMTS-13 activity were seen at VWF:Ag levels around $50 \mathrm{U} / \mathrm{dL}$. Postoperative ADAMTS-13 activity and VWF:CB varied considerably at different concentrations of VWF. In addition, the VWF:CB line was considerably flattened after surgery versus baseline (Figure 2B, different slopes, $\mathrm{P}=0.0477$ ), suggesting lowered VWF biological activity relative to its

Table 3. Postoperative changes in laboratory parameters compared with baseline.

\begin{tabular}{lccc}
\hline & Baseline $(\mathrm{n}=45)$ & Postoperative $(\mathrm{n}=45)$ & $\mathrm{P}$ \\
\hline $\mathrm{SpO}_{2}(\%)$ & $80(53-91)$ & $90(71-99)$ & $<0.0001$ \\
Hematocrit $(\%)$ & $47 \pm 7$ & $36 \pm 7$ & $<0.0001$ \\
Hemoglobin $(\mathrm{g} / \mathrm{dL})$ & $15.9 \pm 2.4$ & $11.7 \pm 2.1$ & $<0.0001$ \\
Platelets $\left(\mathrm{x} 10^{9} / \mathrm{L}\right)$ & $317 \pm 116$ & $189 \pm 113$ & $<0.0001$ \\
Leukocytes $\left(\mathrm{cell} / \mathrm{mm}^{3}\right)$ & $10702 \pm 3612$ & $16067 \pm 6826$ & $<0.0001$ \\
VWF:Ag $(\mathrm{U} / \mathrm{dL})$ & $75(51-159)$ & $212(59-327)$ & $<0.0001$ \\
VWF:CB $(\%)$ & $48 \pm 21$ & $94 \pm 33$ & $<0.0001$ \\
ADAMTS-13:Ag $(\mu \mathrm{g} / \mathrm{mL})$ & $0.91 \pm 0.30$ & $0.70 \pm 0.25$ & $<0.0001$ \\
ADAMTS-13 activity $(\%)$ & $64 \pm 25$ & $143 \pm 59$ & $<0.0001$ \\
VWF:Ag/ADAMTS-13 activity & $1.20(0.67-4.84)$ & $1.54(0.51-5.13)$ & 0.0029 \\
VWF multimers & $0.44(0.11-0.56)$ & $0.45(0.17-0.63)$ & 0.5360 \\
\hline
\end{tabular}

Data are reported as means \pm SD or median and range. ADAMTS-13:Ag = plasma ADAMTS-13 antigenic concentration; $\mathrm{SpO}_{2}=$ peripheral oxygen saturation; VWF:Ag = plasma von Willebrand factor antigenic concentration; VWF:Ag/ADAMTS- 13 activity = ratio of VWF antigenic concentration to ADAMTS-13 activity; VWF:CB = plasma VWF activity reported as collagen binding capacity; VWF multimers = ratio of low molecular weight multimer density to total multimer density of VWF. Data were collected at $48 \mathrm{~h}$ postoperatively from 45 patients who were alive at that time. The Wilcoxon test and the Student $t$-test for pairwise observations were used for statistical analysis. 
antigenic concentration.

Preoperative levels of six specific variables (VWF:Ag, VWF:CB, ADAMTS-13:Ag, ADAMTS-13 activity, VWF:Ag/ ADAMTS-13 activity ratio, and the relative density of low molecular weight VWF multimers) were tested for a possible association with postoperative bleeding. In univariate analysis, only preoperative ADAMTS-13 activity was significantly (negatively) associated with bleeding $(P=0.0265)$. The hazard ratio associated with levels of ADAMTS-13 activity below the default (mean) level of $64.6 \%$ was 11.33 $(95 \% \mathrm{Cl}=1.33-96.81)$. After testing for possible confounders and analyzing other variables, such as demographic data, oxygen saturation, coagulation tests, the need for cardiopulmonary bypass and its duration, and first operation versus reoperation, the final multivariate model included three variables potentially associated with postoperative bleeding, namely a low preoperative ADAMTS-13 activity, APTT and the need for ON-pump surgery. Significant postoperative bleeding occurred in $40 \%$ of patients with a preoperative ADAMTS-13 activity of $<64.6 \%$ (versus $6 \%$ of patients above this level, $\mathrm{P}=0.0170$ ), $39 \%$ of patients undergoing ON-pump surgery (versus 6\% of those undergoing OFF-pump procedures, $P=0.0180)$, and $55 \%$ of patients with both risk factors (versus $7 \%$ of individuals with one or none of them, $\mathrm{P}=0.0010)$.

For 3 patients who died postoperatively, preoperative levels of the biochemical parameters were within the range of the remaining patients. The multimeric structure of VWF was similar to the one shown as baseline in Figure 1. One of these patients (univentricular heart, 5.5 years of age) had postoperative bleeding, with a preoperative ADAMTS-13 activity of $59 \%$.

\section{Discussion}

The congenital heart disease patients in this study had a common profile, namely hypoxemia and polycythemia combined with decreased ADAMTS-13 and VWF:Ag levels. Degradation of VWF multimers was demonstrated in the majority of patients, suggesting abnormal interaction of the VWF protein with membranes (platelets, endothelium) followed by enzymatic cleavage. Therefore, decreased ADAMTS-13 activity (associated with a mild decrease in ADAMTS-13:Ag) was likely a result of chronic enzyme consumption, although decreased production cannot be excluded (the ADAMTS-13 antigen was directly related to peripheral oxygen saturation). We speculate that in patients with CCHD, abnormal interactions of VWF with membranes may occur at sites of altered flow conditions (for example, systemic-to-pulmonary connections, either normally existing or surgically created). Although it has been acknowledged that $A B O$ blood groups influence the circulating levels of VWF, with lower antigenic concentration being expected in group "O" subjects (22), this does not seem to be the main reason for decreased VWF in the present study, since low VWF:Ag was detected even in "nongroup O" individuals. In this study, ADAMTS-13 levels were not influenced by ABO blood groups.

The changes in laboratory parameters observed postoperatively resemble those seen in conditions associated with acute phase (inflammatory) reaction $(10,11,23)$. The intensity of these changes, however, varies considerably depending on the severity of the underlying disease. In sepsis with organ failure, cirrhosis and advanced liver dysfunction, importantly elevated VWF:Ag has been reported in association with decreased ADAMTS-13 activity, resulting in a marked increase in VWF:Ag/ADAMTS-13 activity ratio. In these instances,

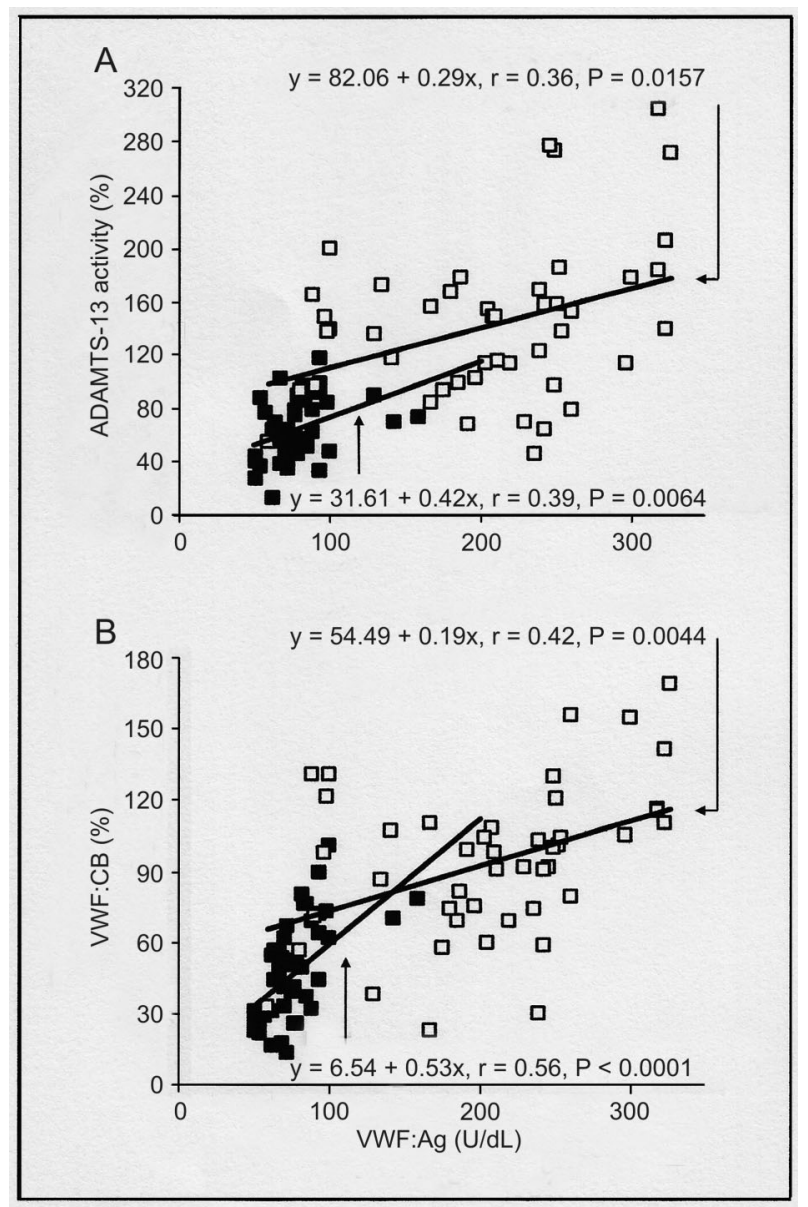

Figure 2. Preoperative (filled squares) and postoperative (open squares) correlations of plasma ADAMTS-13 activity $(A)$ and von Willebrand factor activity $(B, V W F: C B)$ with VWF antigenic concentration (VWF:Ag) in children with cyanotic congenital heart disease. Preoperative and postoperative mean values of ADAMTS-13 activity adjusted for VWF:Ag were 84 and 122\%, respectively ( $P=0.0066$, ANCOVA). In $B$, the slopes are different $(P=0.0477)$, indicating lowered $V W F: C B$ relative to VWF:Ag. Preoperative and postoperative adjusted mean values of VWF:CB were 62 and $79 \%$, respectively $(P=0.0395$, ANCOVA). 
proteolysis of VWF becomes relatively reduced, and ultralarge VWF multimers are seen in the circulation in association with thrombocytopenia. Enzyme consumption and decreased synthesis likely account for the negative correlation of ADAMTS-13 activity with VWF:Ag $(11,24)$. In the less severe situation of preeclampsia, increased VWF:Ag and unchanged (not decreased) ADAMTS-13 activity have been observed in the absence of ultralarge VWF multimers or thrombocytopenia (9). The postoperative changes observed in this study were not as dramatic as those reported in sepsis. We observed an important elevation of VWF:Ag with a mild increase in VWF:CB relative to baseline and continued cleavage of the VWF protein. In contrast to sepsis, ADAMTS-13 activity was increased. However, the VWF:Ag/ADAMTS-13 ratio was elevated compared with the preoperative value, and ADAMTS-13:Ag was actually decreased. We speculate that a fraction of ADAMTS-13 protein was converted to the active form, thus explaining an instantaneous increase in the protease activity even in the presence of total ADAMTS-13 decline. Ultralarge VWF multimers were not present in any patients, suggesting that the metalloprotease activity was sufficient to prevent disseminated thrombosis (there was a decreased platelet count, but not thrombocytopenia). Mannucci et al. (16) showed decreased ADAMTS-13 activity (and increased VWF:Ag) in adults undergoing cardiac surgery. The postoperative increase in the active/activatable fraction of VWF protease (relative to its antigenic concentration) observed here may be a characteristic of the pediatric population with CCHD undergoing surgery, although we must consider that we did not measure biochemical parameters immediately after surgery as done by the other investigators.

Many substances and conditions are known to cause endothelial cell activation and release of VWF from Weibel-Palade bodies, including hypoxia, epinephrine, thrombin, fibrin, cytokines, endotoxin, components of the

\section{References}

1. Uemura $M$, Tatsumi $K$, Matsumoto $M$, Fujimoto $M$, Matsuyama $\mathrm{T}$, Ishikawa $\mathrm{M}$, et al. Localization of ADAMTS13 to the stellate cells of human liver. Blood 2005; 106: 922-924, doi: 10.1182/blood-2005-01-0152.

2. Zhou W, Inada M, Lee TP, Benten D, Lyubsky S, Bouhassira EE, et al. ADAMTS13 is expressed in hepatic stellate cells. Lab Invest 2005; 85: 780-788, doi: 10.1038/ labinvest. 3700275 .

3. Turner N, Nolasco L, Tao Z, Dong JF, Moake J. Human endothelial cells synthesize and release ADAMTS-13. J Thromb Haemost 2006; 4: 1396-1404, doi: 10.1111/j.15387836.2006.01959.x.

4. Tsai HM. Von Willebrand factor, ADAMTS13, and thrombotic thrombocytopenic purpura. $J$ Mol Med 2002; 80: 639647, doi: 10.1007/s00109-002-0369-8.

5. Tsai HM. ADAMTS13 and microvascular thrombosis. Expert Rev Cardiovasc Ther 2006; 4: 813-825, doi: 10.1586/ complement system, and reactive oxygen intermediates (25-31). Many of these elements are probably present in the postoperative scenario of CCHD, and might explain the elevation of plasma VWF:Ag. Although hepatic stellate cell damage may be considered as a potential cause of altered ADAMTS-13 level $(32,33)$, our postoperative findings are probably better explained by moderate protease consumption in the presence of elevated VWF:Ag. Altered synthesis (cytokinemia) and/ or abnormal degradation (plasmin, thrombin) cannot be excluded $(34,35)$.

Previously, preoperative abnormalities of VWF structure have been associated with postoperative bleeding in patients with aortic valve stenosis. Altered flow conditions through the aortic valve probably account for unfolding of the VWF molecule, thus facilitating its binding to platelet membrane glycoproteins, with subsequent proteolytic cleavage (36). Our data do not necessarily indicate a causal relationship between lowered preoperative ADAMTS-13 activity and postoperative bleeding. Rather, altered ADAMTS-13 levels should be viewed as part of an overall process of microvascular dysfunction, where abnormal functioning of endothelial cells and platelets, as well as inflammatory mediators contribute to an increased risk of bleeding. Further studies are necessary to clarify these intriguing and interesting relationships.

Thus, children with CCHD have abnormalities in circulating ADAMTS-13 and its substrate VWF that resemble those observed in disorders associated with microangiopathy and coagulopathy. Preoperative and postoperative data suggested abnormal proteolytic cleavage of VWF multimers associated with ADAMTS-13 consumption.

\section{Acknowledgments}

Research supported by FAPESP (\#05/59890-9).

14779072.4.6.813.

6. Feys HB, Canciani MT, Peyvandi F, Deckmyn $H$, Vanhoorelbeke K, Mannucci PM. ADAMTS13 activity to antigen ratio in physiological and pathological conditions associated with an increased risk of thrombosis. $\mathrm{Br} \mathrm{J}$ Haematol 2007; 138: 534-540, doi: 10.1111/j.1365-2141. 2007.06688.x.

7. Crawley JT, Lane DA, Woodward M, Rumley A, Lowe GD. Evidence that high von Willebrand factor and low ADAMTS13 levels independently increase the risk of a non-fatal heart attack. J Thromb Haemost 2008; 6: 583-588, doi: 10.1111/j. 1538-7836.2008.02902.x.

8. Bongers TN, de Bruijne EL, Dippel DW, de Jong AJ, Deckers JW, Poldermans D, et al. Lower levels of ADAMTS13 are associated with cardiovascular disease in young patients. Atherosclerosis 2009; 207: 250-254, doi: 10.1016/j.atherosclerosis.2009.04.013. 
9. Molvarec A, Rigo J Jr, Boze T, Derzsy Z, Cervenak L, Mako $\mathrm{V}$, et al. Increased plasma von Willebrand factor antigen levels but normal von Willebrand factor cleaving protease (ADAMTS13) activity in preeclampsia. Thromb Haemost 2009; 101: 305-311.

10. Hyun J, Kim HK, Kim JE, Lim MG, Jung JS, Park S, et al. Correlation between plasma activity of ADAMTS-13 and coagulopathy, and prognosis in disseminated intravascular coagulation. Thromb Res 2009; 124: 75-79, doi: 10.1016/ j.thromres.2008.11.020.

11. Claus RA, Bockmeyer CL, Budde U, Kentouche K, Sossdorf $M$, Hilberg $T$, et al. Variations in the ratio between von Willebrand factor and its cleaving protease during systemic inflammation and association with severity and prognosis of organ failure. Thromb Haemost 2009; 101: 239-247.

12. Zonis Z, Seear M, Reichert C, Sett S, Allen C. The effect of preoperative tranexamic acid on blood loss after cardiac operations in children. J Thorac Cardiovasc Surg 1996; 111: 982-987, doi: 10.1016/S0022-5223(96)70374-4

13. Turner-Gomes SO, Nitschmann EP, Norman GR, Andrew ME, Williams WG. Effect of heparin loading during congenital heart operation on thrombin generation and blood loss. Ann Thorac Surg 1997; 63: 482-488, doi: 10.1016/S0003-4975(96)01215-5.

14. Levin E, Wu J, Devine DV, Alexander J, Reichart C, Sett S, et al. Hemostatic parameters and platelet activation marker expression in cyanotic and acyanotic pediatric patients undergoing cardiac surgery in the presence of tranexamic acid. Thromb Haemost 2000; 83: 54-59.

15. Lill MC, Perloff JK, Child JS. Pathogenesis of thrombocytopenia in cyanotic congenital heart disease. Am J Cardiol 2006; 98: 254-258, doi: 10.1016/j.amjcard.2006.01.083.

16. Mannucci PM, Parolari A, Canciani MT, Alemanni F, Camera M. Opposite changes of ADAMTS-13 and von Willebrand factor after cardiac surgery. J Thromb Haemost 2005; 3: 397-399, doi: 10.1111/j.1538-7836.2005.01115.x.

17. Lo B, Nierich AP, Kalkman CJ, Fijnheer R. Relatively increased von Willebrand factor activity after off-pump coronary artery bypass graft surgery. Thromb Haemost 2007; 97: 21-26.

18. Panzer S, Badr ER, Schneller A, Kaider A, Koren D, Eichelberger $B$, et al. Loss of high-molecular-weight von Willebrand factor multimers mainly affects platelet aggregation in patients with aortic stenosis. Thromb Haemost 2010; 103: 408-414, doi: 10.1160/TH09-06-0391.

19. Lopes AA, Soares RP, Maeda NY. A mathematical framework for group analysis of von Willebrand factor multimeric composition following luminography. Braz J Med Biol Res 2002; 35: 1259-1263, doi: 10.1590/S0100879X2002001100002.

20. Siekmann J, Turecek PL, Schwarz HP. The determination of von Willebrand factor activity by collagen binding assay. Haemophilia 1998; 4 (Suppl 3): 15-24, doi: 10.1046/j.13652516.1998.0040s3015.x.

21. Rick ME, Moll S, Taylor MA, Krizek DM, White GC, Aronson DL. Clinical use of a rapid collagen binding assay for von Willebrand factor cleaving protease in patients with thrombotic thrombocytopenic purpura. Thromb Haemost 2002; 88: 598-604.

22. Franchini M, Capra F, Targher G, Montagnana M, Lippi G. Relationship between $\mathrm{ABO}$ blood group and von Willebrand factor levels: from biology to clinical implications. Thromb $\mathrm{J}$ 2007; 5: 14, doi: 10.1186/1477-9560-5-14.

23. Mannucci PM, Canciani MT, Forza I, Lussana F, Lattuada A, Rossi E. Changes in health and disease of the metalloprotease that cleaves von Willebrand factor. Blood 2001; 98: 2730-2735, doi: 10.1182/blood.V98.9.2730.

24. Kremer Hovinga JA, Zeerleder S, Kessler P, Romani de WT, van Mourik JA, Hack CE, et al. ADAMTS-13, von Willebrand factor and related parameters in severe sepsis and septic shock. J Thromb Haemost 2007; 5: 2284-2290, doi: 10.1111/j.1538-7836.2007.02743.x

25. Pinsky DJ, Naka Y, Liao H, Oz MC, Wagner DD, Mayadas TN, et al. Hypoxia-induced exocytosis of endothelial cell Weibel-Palade bodies. A mechanism for rapid neutrophil recruitment after cardiac preservation. J Clin Invest 1996; 97: 493-500, doi: 10.1172/JCl118440.

26. Zavoico GB, Ewenstein BM, Schafer AI, Pober JS. IL-1 and related cytokines enhance thrombin-stimulated PGI2 production in cultured endothelial cells without affecting thrombin-stimulated von Willebrand factor secretion or platelet-activating factor biosynthesis. J Immunol 1989; 142: 3993-3999.

27. Ribes JA, Francis CW, Wagner DD. Fibrin induces release of von Willebrand factor from endothelial cells. J Clin Invest 1987; 79: 117-123, doi: 10.1172/JCl112771.

28. Schorer AE, Moldow CF, Rick ME. Interleukin 1 or endotoxin increases the release of von Willebrand factor from human endothelial cells. Br J Haematol 1987; 67: 193197, doi: 10.1111/j.1365-2141.1987.tb02326.x.

29. Paleolog EM, Crossman DC, McVey JH, Pearson JD. Differential regulation by cytokines of constitutive and stimulated secretion of von Willebrand factor from endothelial cells. Blood 1990; 75: 688-695.

30. Hattori R, Hamilton KK, McEver RP, Sims PJ. Complement C5b-9 stimulates von Willebrand factor secretion from human endothelium. Circulation 1988; 78: II-117, (Abstract).

31. Vischer UM, Jornot L, Wollheim CB, Theler JM. Reactive oxygen intermediates induce regulated secretion of von Willebrand factor from cultured human vascular endothelial cells. Blood 1995; 85: 3164-3172.

32. Jarrar $\mathrm{D}$, Chaudry $\mathrm{IH}$, Wang $\mathrm{P}$. Organ dysfunction following hemorrhage and sepsis: mechanisms and therapeutic approaches (Review). Int J Mol Med 1999; 4: 575-583.

33. Kume $\mathrm{Y}$, Ikeda H, Inoue M, Tejima K, Tomiya T, Nishikawa $\mathrm{T}$, et al. Hepatic stellate cell damage may lead to decreased plasma ADAMTS13 activity in rats. FEBS Lett 2007; 581: 1631-1634, doi: 10.1016/j.febslet.2007.03.029.

34. Cao WJ, Niiya M, Zheng XW, Shang DZ, Zheng XL. Inflammatory cytokines inhibit ADAMTS13 synthesis in hepatic stellate cells and endothelial cells. J Thromb Haemost 2008; 6: $1233-1235$, doi: $10.1111 / \mathrm{j} .1538-$ 7836.2008.02989.x.

35. Crawley JT, Lam JK, Rance JB, Mollica LR, O'Donnell JS, Lane DA. Proteolytic inactivation of ADAMTS13 by thrombin and plasmin. Blood 2005; 105: 1085-1093, doi: 10.1182/ blood-2004-03-1101.

36. Pareti Fl, Lattuada A, Bressi C, Zanobini M, Sala A, Steffan $A$, et al. Proteolysis of von Willebrand factor and shear stress-induced platelet aggregation in patients with aortic valve stenosis. Circulation 2000; 102: 1290-1295, doi: 10.1161/01.CIR.102.11.1290. 